\title{
Using Multiple Path-Constraint Mobile Sinks for Energy Efficient Data Collection in Wireless Sensor Networks
}

\author{
P.Majal Stepa Shiny \\ PG Scholor,Department of Information Technology, \\ Regional Center Anna University, Coimbatore
}

\begin{abstract}
Data collection is a fundamental task of WSN. It aims to collect sensor readings in a sensory field through sinks for analysis and processing. Research has shown that sinks deplete most of the battery power for collecting the data than processing it. Nonuniform energy consumption causes degraded network performance and shortens network lifetime. Recently, sink mobility with limited path has been exploited to reduce and balance the energy expenditure among sensors. This paper address this issue and propose a new data collection scheme, which enhance the Maximum Amount Shortest Path (MASP) for Multiple Mobile Sinks. This scheme increases network throughput and streamlining the energy consumption by optimizing the assignment of sensor nodes to subsinks and subsinks to mobile sinks, using Genetic Algorithm. An existing two-phase communication protocol designed for single mobile sink is enhanced to support multiple mobile sinks. The proposed algorithms and protocols are validated through simulation experiments using NS2.
\end{abstract}

Keywords- Wireless Sensor Networks ( WSN), Maximum Amount Shortest Path(MASP), Mobile sink,Subsink,Direct Communication Area(DCA) ,Multihop Communication Area(MCA)

\section{INTRODUCTION}

Wireless Sensor Networks (WSNs) have been widely considered as one of the most important technologies for the twenty-first century. A WSN typically consists of a large number of lowpriced, low-power, and multi- functional sensor nodes that are deployed in a region of significance. These sensor nodes are small in size, but are equipped with sensors, implant microprocessors, and radio transceivers, and therefore have not only sensing capacity, but also data handling and communicating capability. They communicate over a short distance via a wireless medium and work together to complete a common task, for example, environment monitor, battlefield observation, and industrial process control. Reducing energy utilization is the most important objective in the design of a sensor network. Sensor nodes are power-driven by battery and it is often very difficult or even impossible to change or renew their batteries, it is crucial to reduce the power use of sensor nodes so that the lifetime of the sensor nodes, as well as the whole network is extended.

\section{RELATED WORK}

In [1],[2] the mobile sinks are fixed on vehicles or animals moving randomly to collect information that are sensed by the sensor node .It also analyses architecture to collect sensor data in sparse sensor networks. This approach exploits the presence of mobile entities (called MULEs) present in the surroundings.
MULEs accept data from the sensor in secure range, safeguard it, and drop off the data to wired access points.

In [3],[4], path constrained sink mobility is used to improve the energy efficiency in sensor networks using single hop which may be infeasible because of the limits of location and communication power. A routing protocol called MobiRoute is proposed in [7] for Wireless Sensor Networks with a path predictable mobile sink where all sensor nodes need to be aware of the movement of the mobile sink. In [3],[4],[6] various data collection problems are concerned to improve the performance of the network. In [5], path constrained sink mobility is discussed to improve the energy efficiency of multihop sensor networks using a single mobile sink. They have also contains the coding for MASP for single mobile sink. In this paper a code is developed for multiple mobile sink to increase the amount of gathered data and decrease the energy consumption.

\section{SYSTEM ARCHITECTURE}

Based on the communication area of the mobile sinks the coverage area can be divided into two parts ,the Direct Communication Area (DCA) and Multihop Communication area (MCA).Sensor nodes that presents inside the DCA are called subsinks, which can directly transmit data to the mobile sinks because they are close to the trajectory of mobile sinks. And the sensor nodes residing within the MCA are called members, transmit the data to the mobile sink through subsinks(sensor to subsink and subsink to mobile sink)

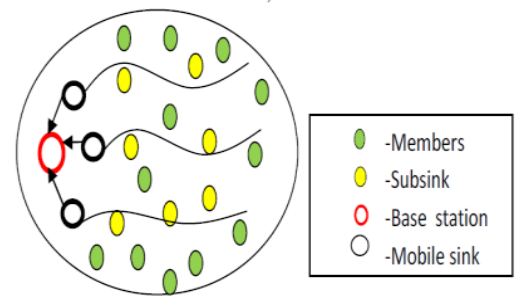

Fig 1: Topology Creation

\section{BI-STAGE DATA COLLECTION PROTOCOL}

A Bi-stage data collection protocol is proposed in this chapter which includes efficient subsink discovery and data collection procedures. It consist of two main phases: Discover phase and Data Collection phase.

\subsection{Discover Phase}

The main job of discover phase is assigning the members to their corresponding subsink and subsink to their corresponding mobile sink.It consist of three steps. 


\section{Step1:}

In this step all the mobile sinks transmit the broadcast messages continuously. The nodes that are receiving the broadcast messages from the mobile sink are automatically selected as the subsink of the corresponding mobile sink. Then the SPT(Shortest Path Tree) is developed fot the entire network.

\section{Step2:}

In this step the subsinks send the shortest hop information collected in step 1 to their corresponding mobile sink when it passing it.This information is enough for MASP calculation.Finally Gentic algorithm (In section 6) is executed to obtain the optimized assignment between all subsink and mobile sink and all members to subsink.

\section{Step 3:}

In step 3,All the mobile sinks traverse the path again to broadcast the results of the member and subsink assignment.

\subsection{Data Collection phase:}

In this phase ,all nodes start collecting data from the sensed area.The members send the data to their subsink and the subsink send the data to their mobile sink. The mobile sinks send the collected data to the base station for processing it.

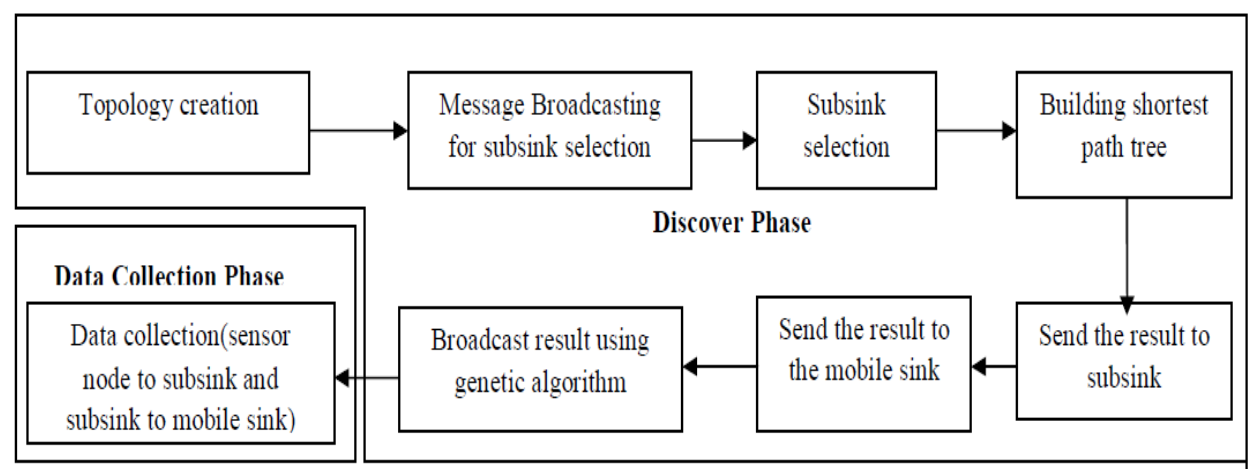

Fig 2: Bi-stage data collection protocol

\section{MASP FOR MULTIPLE SINKS}

The amount of data transmitted to the Base station by a mobile sink in one round consist of the data collected from all mobile sinks as follows:

$$
q_{\text {total }}=\sum_{i=1}^{n_{m s}} q_{i}
$$

where $\mathrm{q}_{\mathrm{i}}$ is the amount of data collected by each mobile sink i per round and $\mathrm{n}_{\mathrm{ms}}$ is the number of mobile sink. The amount of data collected by the mobile sink consists of the data collected from all its subsinks.

$$
q_{i}=\sum_{i=1}^{n_{s}} s_{i}
$$

where $S_{i}$ is the amount of data collected by each subsink associated with a single mobile sink and $\mathrm{n}_{\mathrm{s}}$ is the number of subsinks.

Let $r_{i}$ members be assigned to the subsink $i$ and each member collect data with a rate of $d_{s}$ and transmit to subsink continuously for $\mathrm{t}$ seconds. Also assume that the subsink $\mathrm{i}$ transmit data to the mobile sink for the duration of $t_{i}$ with the data rate of $d_{t}$ per round . Then the amount of data $q_{i}$ is calculated as follows

$$
s_{i}=\min \left[d_{t} t_{i},\left(d_{s} t\right) r_{i}\right]
$$

The main objective of this project is to maximize the total amount of collected by mobile sinks per round. Actually a $\mathrm{q}_{\text {total }}$ calculation is related to the node density of a network. As the number of nodes in the monitored area is high (highly densed), it is difficult to collect data from all the sensor nodes within the communication time. When the number of nodes in monitored area reduced (lightly densed) it is possible to collect more data. Here we develop a new scheme to find if a network density is high or low. Let $\mathrm{K}_{\mathrm{i}}$ subsinks be assigned to the mobile sink $\mathrm{i}$ and each subsink collect the data at the rate of $\mathrm{d}_{\mathrm{k}}$ and transmit to the mobile sink continuously for $\mathrm{t}_{1 \mathrm{i}}$ seconds, then

$$
k_{i}^{m}=\frac{d_{k} t_{1 i}}{d_{t} t_{i}} \text { and } r_{i}^{m}=\frac{d_{t} t_{i}}{d_{s} t}
$$

Then we can say that the network density is high if

$$
n_{m} \geq \sum_{i=1}^{n_{s}} r_{i}^{m} \text { and } n_{s} \geq \sum_{i=1}^{n_{m s}} k_{i}^{m}
$$

And the density of a network is low if

$$
n_{m}<\sum_{i=1}^{n_{s}} r_{i}^{m} \text { and } n_{s}<\sum_{i=1}^{n_{m s}} k_{i}^{m}
$$

We have some conditions that must be satisfied to maximize the total amount of data collected by all mobile sink,

$r_{i} \geq r_{i}^{m}$ and $k_{i} \geq k_{i}^{m} \forall \mathrm{i} \quad, \quad$ for high density network. 
$r_{i}<r_{i}^{m}$ and $\quad k_{i}<k_{i}^{m} \forall \mathrm{i}, \quad$ for low density network.

where $r_{i}^{m}$ is called as Minimum or Maximum requirement on the number of members assigned to the sub sink. $\mathrm{K}_{\mathrm{i}}^{\mathrm{m}}$ is called as minimum or maximum requirement on the number of sub sink assigned to the mobile sink. In order to maximize the total amount of data in low density network, it must be guaranteed that no sub sink owns more members than its Minimum or Maximum requirements $\left(\mathrm{r}_{\mathrm{i}}^{\mathrm{m}}\right)$ and no mobile sink owns more sub sink than its Minimum or Maximum requirements $\left(\mathrm{k}_{\mathrm{i}}^{\mathrm{m}}\right)$.

Based on the above analysis, our optimization problem is formulated as follows: $\quad r_{i} \geq r_{i}^{m}, \forall i=1 \ldots . . n_{s}$ if $n_{m} \geq \sum_{i=1}^{n_{s}} r_{i}^{m}$ and

$$
k_{i} \geq k_{i}^{m}, \forall i=1 \ldots \ldots . . n_{m s} n_{s} \geq \sum_{i=1}^{n_{s}} k_{i}^{m}
$$

$$
\begin{aligned}
& r_{i} \leq r_{i}^{m}, \forall i=1 \ldots \ldots . n_{s} \quad \text { if } \quad n_{m} \leq \sum_{i=1}^{n_{s}} r_{i}^{m} \\
& k_{i} \leq k_{i}^{m}, \forall i=1 \ldots \ldots . n_{m s \text { if }} n_{s} \leq \sum_{i=1}^{n_{s}} k_{i}^{m}
\end{aligned}
$$

In our problem, each sub sink is assigned to only one mobile sink and each mobile sink has its own requirement on the number of its subsink likewise each member is assigned to only one sub sink, and each sub sink has its own requirement on the number of its members.

Let us consider the following terminology:

- A:a matrix consisting of binary elements $a_{i j}$, for $\mathrm{i}=1, \ldots \ldots . ., \mathrm{n}_{\mathrm{s}}$ and $\mathrm{j}=1, \ldots \ldots ., \mathrm{n}_{\mathrm{ms}}$, where

$\mathrm{a}_{\mathrm{ij}} \int 1, \quad$ if subsink $\mathrm{i}$ is assigned to mobile sink $\mathrm{j}(11)$

$0, \quad$ otherwise elements $b_{i j}$ for $i=1, \ldots \ldots ., n_{m}$ and $\mathrm{j}=1, \ldots \ldots \ldots \ldots . . ., \mathrm{n}_{\mathrm{s}}$, where

$$
\mathrm{b}_{\mathrm{ij}}=\{1, \quad \text { if node } \mathrm{i} \text { is assigned to subsink } \mathrm{j}
$$$$
0, \quad \text { otherwise }
$$

- $\mathrm{H}: \mathrm{a}$ matrix consisting of $\mathrm{h}_{\mathrm{ij}}$, for $\mathrm{i}=1, \ldots \ldots, \mathrm{n}_{\mathrm{s}}$ and $j=1, \ldots \ldots ., n_{m s}$, where $h_{i j}$ denotes the number of hops from subsink i to mobilesink $\mathrm{j}$.

- L: a matrix consisting of $1_{i j}$, for $\mathrm{i}=1, \ldots \ldots, \mathrm{n}_{\mathrm{m}}$ and $\mathrm{j}=1, \ldots \ldots . ., n_{\mathrm{s}}$, Where $\mathrm{l}_{\mathrm{ij}}$ denotes the number of hops from member $i$ to subsink $\mathrm{j}$.

The MASP optimization problem can be converted to a $0-1$ Integer Linear Programming as follows:
$\min \sum_{i=1}^{n_{s}} \sum_{j=1}^{n_{m s}} a_{i j} h_{i j}$
$\min \sum_{i=1}^{n_{s}} \sum_{j=1}^{n_{m s}} b_{i j} l_{i j}$
$\sum_{j=1}^{n_{m s}} a_{i j}=1$
$\forall i$
and $\sum_{j=1}^{n_{s}} b_{i j}=1 \quad \forall i$

\section{GENETIC ALGORITHM FOR MULTIPLE MOBILE SINK}

\subsection{Initial Population}

Initially we have to assume each member as the chromosome based on the positions. Then we have to assign each member to the subsink based on $r_{i}{ }^{m}$. Likewise the subsinks are assigned to the mobile sink randomly satisfy the constraint (14).

\subsection{Fitness Function}

The fitness function determines the possible solutions passed on to multiply and mutate into the next generation of solutions. This is usually done by analyzing the chromosome which hold some data about a particular solution to the problem we are trying to solve. The fitness function will look at the chromosome and make some qualitative assessment, returning a fitness value for that solution. A solution with good fitness value will be accepted as input to the rest of the genetic algorithm and the solutions with low fitness value will be eliminated from the process.

The fitness of the solution is calculated as follows:

$$
\mathrm{f}(\mathrm{A})=\sum_{i=1}^{n_{s}} \sum_{j=1}^{n_{m s}} a_{i j} h_{i j} \sum_{i=1}^{n_{m}} b_{i j} l_{i j}
$$

$\mathrm{n}_{\mathrm{s}} \quad$-Number of Subsink

$\mathrm{n}_{\mathrm{m}} \quad$-Number of Members

$\mathrm{a}_{\mathrm{ij}} \quad$-A Matrix that describes the relationship between subsink and mobile sink.

$b_{i j} \quad-A$ Matrix that describes the relationship between members and sub sink.

$\mathrm{h}_{\mathrm{ij}} \quad$-A Matrix describes the hopcount between subsink and mobile sink

$\mathrm{l}_{\mathrm{ij}} \quad$ A Matrix describes the hopcount between members and sub sink

Higher fitness means more energy consumption. So our aim is to reduce the fitness value to decrease the energy consumption. 


\subsection{Crossover}

This operator randomly chooses a locus and exchanges the subsequences before and after that locus between two chromosomes to create two offspring. For example, the strings 10000100 and 11111111 could be crossed over after the third locus in each to produce the two offspring 10011111 and 11100100. The crossover operator roughly mimics biological recombination between two single-chromosome organisms

\subsubsection{Algorithm: Crossover operator based on the unfitness value for each member $i$ subsink $j$ and mobile sink $k$}

Initialize temporary variables $\mathrm{v}_{1}, \mathrm{v}_{2}, \mathrm{v}_{3} \ldots \ldots \mathrm{v}_{\mathrm{n}}$ to 0 and $\mathrm{x}_{1}, \mathrm{x}_{2}, \mathrm{x}_{3} \ldots . \mathrm{x}_{\mathrm{n}}$ to 0

for $\left(j=1\right.$ to $n_{m s}, x j=a_{i j}{ }^{p 1}$ and $\left.a_{i j}{ }^{p 2}\right)$

for $\left(\mathrm{j}=1\right.$ to $\mathrm{n}_{\mathrm{s}}, \mathrm{v}_{\mathrm{j}}=\mathrm{b}_{\mathrm{ij}}{ }^{\mathrm{p}}{ }^{1}$ and $\mathrm{b}_{\mathrm{ij}}{ }^{\mathrm{p}}$ )

$$
\begin{aligned}
& \text { if } \sum_{k=1}^{n_{m s}} x_{k}=1 \\
& \text { if } \sum_{k=1}^{n_{s}} v_{k}=1 \\
& \quad \mathrm{~b}_{\mathrm{ij}}{ }^{\mathrm{c}}=\mathrm{b}_{\mathrm{ij}}{ }^{\mathrm{p} 1} \quad \mathrm{j}=1,2,3, \ldots \ldots . \mathrm{n}_{\mathrm{s}}
\end{aligned}
$$

else

$\mathrm{b}_{\mathrm{ij}}{ }^{\mathrm{c}}=\mathrm{b}_{\mathrm{ij}}{ }^{\mathrm{p}}$ with probability $\mathrm{U}\left(\mathrm{BP}_{2}\right) / \mathrm{U}\left(\mathrm{BP}_{1}\right)+$ $\mathrm{U}\left(\mathrm{BP}_{2}\right), \mathrm{j}=1,2,3, \ldots \ldots . \mathrm{n}_{\mathrm{s}}$

$\mathrm{b}_{\mathrm{ij}}{ }^{\mathrm{c}}=\mathrm{b}_{\mathrm{ij}}{ }^{\mathrm{p} 2}$ with probability $\mathrm{U}\left(\mathrm{BP}_{1}\right) / \mathrm{U}\left(\mathrm{BP}_{1}\right)+$ $\mathrm{U}\left(\mathrm{BP}_{2}\right), \mathrm{j}=1,2,3, \ldots \ldots . \mathrm{n}_{\mathrm{s}}$

end if

$$
\mathrm{a}_{\mathrm{ij}}{ }^{\mathrm{c}}=\mathrm{a}_{\mathrm{ij}}^{\mathrm{p} 1} \quad \mathrm{j}=1,2,3, \ldots \ldots \mathrm{n}_{\mathrm{ms}}
$$

else

$\mathrm{a}_{\mathrm{ij}}{ }^{\mathrm{c}}=\mathrm{a}_{\mathrm{ij}}{ }^{\mathrm{p} 1}$ with probability $\mathrm{U}\left(\mathrm{AP}_{2}\right) / \mathrm{U}\left(\mathrm{AP}_{1}\right)+\mathrm{U}\left(\mathrm{AP}_{2}\right)$, $\mathrm{j}=1,2,3, \ldots \ldots . \mathrm{n}_{\mathrm{ms}}$

$\mathrm{a}_{\mathrm{ij}}{ }^{\mathrm{c}}=\mathrm{a}_{\mathrm{ij}} \mathrm{p}^{2}$ with probability $\mathrm{U}\left(\mathrm{AP}_{1}\right) / \mathrm{U}\left(\mathrm{AP}_{1}\right)+\mathrm{U}\left(\mathrm{AP}_{2}\right)$, $\mathrm{j}=1,2,3, \ldots \ldots . \mathrm{n}_{\mathrm{ms}}$

end if

\subsection{Mutation}

This operator randomly flips some of the bits in a chromosome. For example, the string 00000100 might be mutated in its second position to yield 01000100. Mutation can occur at each bit position in a string with some probability, usually very small

\section{7 . SIMULATION ENVIRONMENT}

Table 1: Simulation Environment

\begin{tabular}{|l|l|}
\hline No. of Nodes & 200 \\
\hline Area Size & $400 \mathrm{~m} \mathrm{X} \mathrm{200} \mathrm{m}$ \\
\hline Mac & 802.11 \\
\hline Traffic Source & CBR \\
\hline Packet Size & $500 \mathrm{bytes}$ \\
\hline Transmit Power & $0.02 \mathrm{mw}$ \\
\hline Receiving Power & $0.01 \mathrm{mw}$ \\
\hline Idle Power & $0.2 \mathrm{mw}$ \\
\hline Initial Energy & $20 \mathrm{~J}$ \\
\hline Transmission Range & $250 \mathrm{~m}$ \\
\hline
\end{tabular}

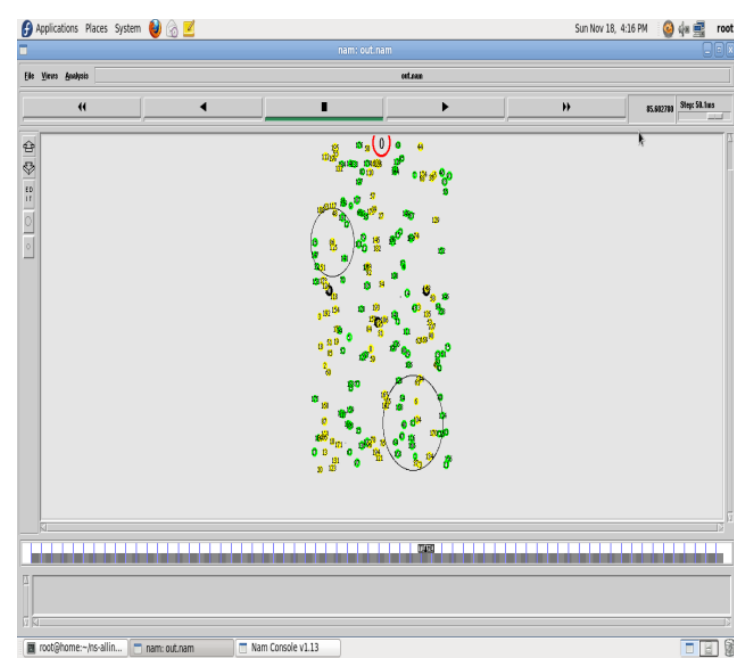

Figure 3: Simulation of wireless sensor network with subsinks

Here node in red color represents the base station, the node in black color represents the mobile sink, node in yellow color represents the subsik and the node in green color represents members.

\subsection{Simulation Result:}

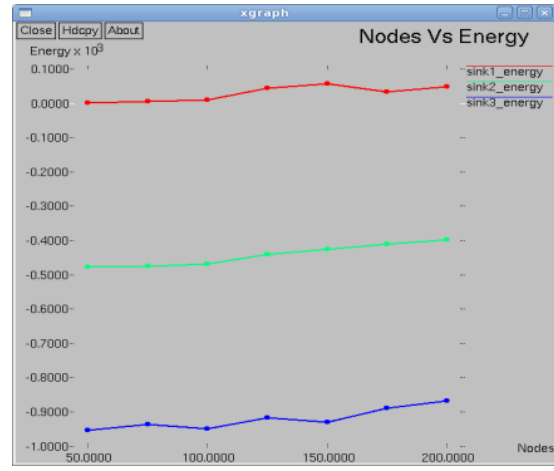

Figure 4: Nodes Vs Energy with multiple sinks 
Figure. 4 shows the relationship between energy consumption and number of sensor nodes that are deployed in the sensing region using MASP with multiple mobile sinks . The curve in red color denotes the energy consumption using single mobile sink. The curve in green color represents the energy consumption using 2 mobile sink. The curve in blue color represents the energy consumption using 3 mobile sink.It clearly shows that the energy consumption is decreased, as the number of mobile sinks increased.

\section{CONCLUSION}

This paper analyzes the performance of the usage of multiple mobile sinks in collecting the data in wireless sensor networks with reduced energy consumption .The Bi-Stage communication protocol is extended to support multiple mobile sinks and its performance is monitored. This work can be enhanced in future work to focus on addressing the various data storage overheads, and packet delivery ratio.

\section{REFERENCES}

[1] R.C. Shah, S. Roy, S. Jain, and W. Brunette, "Data MULEs: Modeling a Three-Tier Architecture for Sparse Sensor Networks," Proc. First IEEE Int'l Workshop Sensor Network Protocols and Applications, pp. 30-41, 2003.

[2] S. Jain, R.C. Shah, W. Brunette, G. Borriello, and S. Roy, "Exploiting Mobility for Energy Efficient Data Collection in Sensor Networks," Mobile Networks and Applications, vol. 11, no. 3, pp. 327-339, 2006.
[3] A. Chakrabarti, A. Sabharwal, and B. Aazhang, "Communication Power Optimization in a Sensor Network with a Path-Constrained Mobile Observer," ACM Trans. Sensor Networks, vol. 2, no. 3, pp. 297-324, Aug. 2006.

[4] L. Song and D. Hatzinakos, "Architecture of Wireless Sensor Networks with Mobile Sinks: SparselyDeployed Sensors," IEEE Trans. Vehicular Technology, vol. 56, no. 4, pp. 1826-1836, July 2007.

[5] Shuai Gao,Hongke Zhang, and Sajal K. Das,"Efficient Data Collection in Wireless Sensor Networks with Path Constrained Mobile Sinks “ IEEE Transactions on Mobile Computing,vol. 10,no.5,pp.592-608,April 2011.

[6] D. Jea, A. Somasundara, and M. Srivastava, "Multiple ControlledMobile Elements (Data Mules) for Data Collection in Sensor Networks," Proc. First IEEE/ACM Int'l Conf. Distributed Computing in Sensor Systems (DCOSS), pp. 244-257, 2005.

[7] A. Kansal, A. Somasundara, D. Jea, M. Srivastava, and D. Estrin, "Intelligent Fluid Infrastructure for Embedded Networks," Proc. ACM MobiSys, pp. 111-124, 2004.

[8] J. Luo, J. Panchard, M. Piorkowski, M. Grossglauser, and J. Hubaux, "MobiRoute: Routing towards a Mobile Sink for Improving Lifetime in Sensor Networks," Proc. Second IEEE/ ACM Int'l Conf. Distributed Computing in Sensor Systems (DCOSS), pp. 480-497, 2006. 